\title{
Quiet PROPELLER MRI Techniques Match the Quality of Conventional PROPELLER Brain Imaging Techniques
}

\author{
I. Corcuera-Solano, A. Doshi, P.S. Pawha, D. Gui, A. Gaddipati, and L. Tanenbaum
}

\begin{abstract}
BACKGROUND AND PURPOSE: Switching of magnetic field gradients is the primary source of acoustic noise in MR imaging. Sound pressure levels can run as high as $120 \mathrm{~dB}$, capable of producing physical discomfort and at least temporary hearing loss, mandating hearing protection. New technology has made quieter techniques feasible, which range from as low as $80 \mathrm{~dB}$ to nearly silent. The purpose of this study was to evaluate the image quality of new commercially available quiet T2 and quiet FLAIR fast spin-echo PROPELLER acquisitions in comparison with equivalent conventional PROPELLER techniques in current day-to-day practice in imaging of the brain.
\end{abstract}

MATERIALS AND METHODS: Thirty-four consecutive patients were prospectively scanned with quiet T2 and quiet T2 FLAIR PROPELLER, in addition to spatial resolution-matched conventional T2 and T2 FLAIR PROPELLER imaging sequences on a clinical 1.5T MR imaging scanner. Measurement of sound pressure levels and qualitative evaluation of relative image quality was performed.

RESULTS: Quiet T2 and quiet T2 FLAIR were comparable in image quality with conventional acquisitions, with sound levels of approximately $75 \mathrm{~dB}$, a reduction in average sound pressure levels of up to $28.5 \mathrm{~dB}$, with no significant trade-offs aside from longer scan times.

CONCLUSIONS: Quiet FSE provides equivalent image quality at comfortable sound pressure levels at the cost of slightly longer scan times. The significant reduction in potentially injurious noise is particularly important in vulnerable populations such as children, the elderly, and the debilitated. Quiet techniques should be considered in these special situations for routine use in clinical practice.

ABBREVIATIONS: $C=$ conventional; $\Delta \mathrm{L}=\mathrm{SPL}$ difference; $\mathrm{Q}=$ quiet; $\mathrm{SPL}=$ sound pressure level

A coustic noise generated during MR imaging contributes to patient discomfort. Problems associated with high levels of acoustic noise include annoyance, anxiety, and verbal communication difficulties between the patient and operator. ${ }^{1,2}$ In addition, the very high noise pressure levels can cause hearing loss. Temporary shifts in hearing thresholds have been reported in $43 \%$ of the patients scanned without ear protection and with improperly fitted earplugs. ${ }^{3}$ In extreme cases, permanent hearing

Received October 8, 2014; accepted after revision December 5.

From the Neuroradiology Section, Department of Radiology (I.C.-S., A.D., P.S.P., L.T.), Icahn School of Medicine at Mount Sinai Hospital, Radiology, New York, New York; and GE Healthcare (D.G., A.G.), Milwaukee, Wisconsin.

Dr Idoia Corcuera-Solano was supported by a grant from the Spanish foundation: Fundación Alfonso Martín Escudero.

Paper previously presented in part at: Annual Meeting of the American Society of Neuroradiology and the Foundation of the ASNR Symposium, May 18-23, 2013; San Diego, California.

Please address correspondence to Lawrence N. Tanenbaum, MD, Mount Sinai Medical Center, Department of Radiology B1234, One Gustave L. Levy Pl,

New York, NY 10029; e-mail: nuromri@gmail.com; @nuromri

http://dx.doi.org/10.3174/ajnr.A4235 impairment can occur. ${ }^{3-5}$ Noise is of particular concern in populations vulnerable to hearing loss such as the very young and elderly and those who may not be able to manage the effectiveness of earplug placement such as patients with psychiatric disorders or reduced levels of consciousness. ${ }^{5}$ Fetal noise exposure is also a concern. ${ }^{6}$

The primary source of acoustic noise in MR imaging procedures is the pulsed currents generated in gradient coils for spatial encoding of the MR signal. ${ }^{7}$ These currents, in the presence of the strong static magnetic field of the MR imaging system, induce significant (Lorentz) forces that cause vibrations in the gradient coils, which, in turn, generate a compression wave in the air perceived as the scanner noise. ${ }^{8-10}$ Previous methods used to ameliorate the high acoustic noise levels of clinical MR imaging include acoustic insulation of the scanner bore, resulting in reduced bore diameter and gradient waveform shaping/filtering ${ }^{11,12}$; bandwidth limiting ${ }^{13}$; and restricting gradient performance-each trading image quality and acquisition speed for only modest noise reduction. More recent studies have demonstrated that innovative pulse-sequence modifications can be applied to achieve 
substantial reductions of acoustic noise while maintaining image quality. Novel, almost silent sequences have recently become available. ${ }^{14-17}$ Before these new techniques can be widely adopted, validation against traditional techniques must be performed.

In this study, we evaluated the image quality of new commercially available quiet T2 PROPELLER (Q-T2) and quiet T2 FLAIR PROPELLER (Q-FLAIR) sequences in comparison with our standard of care conventional T2 PROPELLER (C-T2) and T2 FLAIR PROPELLER (C-FLAIR) techniques. To our knowledge, this is the first study to assess the performance of quiet T2 and quiet T2 FLAIR MR imaging applications in day-to-day clinical practice.

\section{MATERIALS AND METHODS Study Design and Patients}

This prospective single-center study followed the Health Insurance Portability and Accountability Act guidelines, and its protocol was approved by the institutional review board. Written informed consent was obtained from subjects or, in the case of noncompetent patients, their respective parents or next of kin for all procedures, in accordance with the local institutional review board protocol.

Thirty-four consecutive inpatients undergoing routine brain MR imaging between February 2013 and March 2014 were included. Eighteen men and 16 women with a mean age of 54 years (range, 21-96 years) were prospectively enrolled in this intraindividual comparative study and evaluated. All patients were given standard clinical ear protective equipment and underwent brain MR imaging examinations for a variety of clinical purposes. The clinical conditions were stroke $(n=6)$, persistent headache $(n=$ $6)$, demyelinating disease $(n=5)$, mental disorder $(n=3)$, vertigo $(n=2)$, seizure $(n=2)$, brain tumor $(n=3)$, metastatic disease $(n=2)$, infectious disease $(n=2)$, recurrent falls $(n=2)$, and CSF leak $(n=1)$.

\section{MR Imaging Protocol}

MR imaging included all sequences in routine use for each clinical indication, including axial PROPELLER T2 and T2 FLAIR. Added were spatial resolution-matched quiet PROPELLER T2 and quiet T2 FLAIR in the transverse plane. All scans were obtained on a clinical 1.5T MR imaging system (Optima 450W; GE Healthcare, Milwaukee, Wisconsin) by using a 32-channel head coil (24 elements are activated). Section locations were identical across comparison scans. MR imaging data acquisition parameters are summarized in Table 1.

\section{Acoustic Noise Measurements}

Acoustic noise measurements were conducted onsite with the scanner unoccupied by using imaging parameters identical to those in use with patients. The sound pressure level (SPL) values were measured by using a sound level meter (Type 2250; Bruel and Kjaer, Nærum, Denmark), which has an accuracy of $\pm 1 \mathrm{dBA}$, and a microphone (Type 4189; Bruel and Kjaer). The device was placed inside the head coil close to where the ears would be, and measurements were averaged for 20 seconds. The SPL was mea-
Table 1: MR Imaging data-acquisition parameters

\begin{tabular}{lcccc}
\hline & C-T2 & Q-T2 & C-T2 FLAIR & Q-T2 FLAIR \\
\hline TR (ms) & 3066 & 6380 & 9500 & 9500 \\
TE (ms) & 99 & 98 & 190 & 105 \\
TI (ms) & NA & NA & 2250 & 2250 \\
FOV (cm) & $22 \times 22$ & $22 \times 22$ & $23 \times 23$ & $23 \times 23$ \\
Matrix & $320 \times 320$ & $320 \times 320$ & $288 \times 288$ & $288 \times 288$ \\
Section thickness (mm) & 5 & 5 & 5 & 5 \\
Section spacing (mm) & 0 & 0 & 0 & 0 \\
No. of sections & 30 & 30 & 30 & 30 \\
Bandwidth (kHz) & 50 & 41 & 62.5 & 41 \\
Refocus flip angle & $160^{\circ}$ & $160^{\circ}$ & $160^{\circ}$ & $160^{\circ}$ \\
Echo-train length & 28 & 16 & 32 & 18 \\
NEX & 1.7 & 1.5 & 1.7 & 1.5 \\
Acceleration factor & 2 & 2 & 2 & 2 \\
Scan time (min) & 1.26 & 2.16 & 3.10 & 5.20 \\
\hline
\end{tabular}

Note:-NA indicates not applicable.

sured for each sequence by using the same apparatus. We calculated the following parameters:

- SPL difference between conventional and quiet sequences: $\Delta \mathrm{L}=\mathrm{C}-\mathrm{Q}$

- Comparison of power level or sound intensity factor: $\mathrm{I}=$ $10^{\Delta \mathrm{L} / 10}$

- Comparison of sound pressure or sound pressure factor: SP = $10^{\Delta \mathrm{L} / 20}$

- Loudness factor: $\mathrm{LF}=10^{\Delta \mathrm{L} / 33.22}=2^{\Delta \mathrm{L} / 10}$.

\section{Image Quality Assessment}

A qualitative comparison of images acquired with quiet and conventional approaches was performed. Matched image sets were gathered from each patient: Q-T2 $(n=34)$ was compared with C-T2 $(n=34)$; and Q-FLAIR $(n=31)$, with C-FLAIR $(n=31)$. Three patients were not eligible for the T2 FLAIR comparison because both scans were not obtained.

A total of 130 image sets presented in the same order were independently assessed by 2 neuroradiologists (with 10 and 25+ years of experience) blinded to the acquisition technique. The images were evaluated qualitatively on an analysis and viewing workstation (Advantage Workstation 4.6; GE Healthcare) for overall quality and gray matter-white matter differentiation, by using a 3-point scale: 3 being better than expected, 2 being as expected, and 1 being worse than expected. Because alterations in multiecho sequence scan acquisition parameters such as echo spacing and echo-train length can manifest as a blur, perceived blurring (yes/no) was evaluated. All scans benefited from inherent PROPELLER motion resistance; thus, this was not measured. Additionally, readers were asked to rank the 2 pair sets for preference and declare images as better, worse, or equivalent (overall preference). Image quality scores were averaged across both readers for analysis and were presented as mean value and SD.

\section{Statistical Analysis}

Interobserver agreement for assessment of image quality was quantified by weighted $\kappa$ statistics. ${ }^{18}$ A nonparametric paired Wilcoxon test was used to compare the qualitative parameters between the quiet and conventional image pairs. Image quality scores for each set of MR images were analyzed. Qualitative results were expressed as mean and SD. Statistical analysis was performed by using commercially available software (SPSS, Version 20; IBM, 
Armonk, New York). Difference was considered statistically significant at $P<.05$.

\section{RESULTS}

\section{Acoustic Noise Reduction}

The respective average SPL measurements for C-T2, C-FLAIR, and Q-T2 and Q-FLAIR protocols are shown in Table 2. Repeated measurements showed no variation in average and peak SPL; therefore, no SD is reported.

The average SPL difference between C-T2 sequences and Q-T2 was found to be $26.4 \mathrm{~dB}$ ( $26 \%$ reduction). This represents a sound intensity factor difference of 436.5 , a sound pressure factor difference of 21, and a loudness factor difference of 6.2. The SPL difference between C-FLAIR sequences and Q-FLAIR was found to be $28.5 \mathrm{~dB}$ (27\% reduction), which corresponds to differences in a sound intensity factor of 708, a sound pressure factor of 27 , and a loudness factor of 7.2.

\section{Qualitative Analysis}

Interrater reliability was good (Cohen $\kappa$ weighted $=0.67$ ). No significant difference in image quality was found between conventional and quiet sequences $(P>.05)$. Readers expressed no preference between quiet and conventional T2 sequences, and in all cases, gray matter-white matter differentiation and overall quality were rated "as expected." Similarly, image quality scores of the Q-FLAIR and C-FLAIR protocols were comparable. In 1 case, Q-FLAIR was preferred over the conventional technique regarding overall quality, while in another case, C-FLAIR protocol was preferred over Q-FLAIR regarding gray matter-white matter differentiation. In all other cases, the quiet and conventional sets were rated "as expected." When the perceived blurring was assessed, no evidence of blur was noted in any case. Image examples are provided in Figs 1 and 2.

\section{DISCUSSION}

Acoustic noise associated with MR imaging procedures is primarily due to pulsed-current-related vibration of the gradient coil support structure. The sounds made by the scanner vary in volume and tone with the type of pulse sequence performed. ${ }^{5,19}$ The acoustic noise varies due to the alteration of the gradient output (rise time or amplitude) with variation of MR imaging parameters. Noise tends to be enhanced by decreases in section thickness, FOV, TR, and TE. ${ }^{5}$

Noise levels for clinical MR imaging pulse sequences run as high as $100-120 \mathrm{~dB},{ }^{19-22}$ levels capable of causing temporary or even permanent hearing loss if hearing protection is not properly applied.

The commercially available quiet sequences used in this study drastically reduce noise levels to approximately $75 \mathrm{~dB}$, a reduction in average SPL of up to $28.5 \mathrm{~dB}$. Quiet PROPELLER uses a standard 2D modified radial sampling scheme with an acoustic noise model to optimize gradient waveforms. ${ }^{23}$ The $k$-space trajectory and data sampling can be optimized so that gradient steps are smaller than those in conventional PROPELLER, resulting in a scan with noise levels $<6 \mathrm{dBA}$ above ambient levels.

At a penalty of only slightly longer scan times, Q-T2 and QFLAIR provided high-quality images comparable with conventional acquisitions. Spatial-resolution-matched quiet sequences had similar scores for overall quality and gray matter-white matter differentiation without an increase in image blur.

To the best of our knowledge, this is the first report in the literature assessing the performance of quiet T2 and quiet T2 FLAIR PROPELLER imaging in the day-to-day practice of clinical imaging. Our results complement the recent previously reported data regarding the use of new quiet scanning approaches. Pierre et $\mathrm{al}^{15}$ compared, in a group of 10 volunteers, a quiet T2 TSE sequence prototype with its equivalent standard T2 TSE sequence and demonstrated that overall image quality remained above acceptable while reducing SPL. Two other recent studies assessed unique 3D T1-weighted silent scan technology in clinical practice, obtaining similarly acceptable results. ${ }^{16,17}$
Table 2: Comparison of acoustic noise

\begin{tabular}{|c|c|c|c|c|c|c|}
\hline & $\begin{array}{c}\text { Conventional } \\
\text { Avg SPL }\end{array}$ & $\begin{array}{c}\text { Quiet } \\
\text { Avg SPL }\end{array}$ & $\Delta \mathrm{L}=\mathrm{C}-\mathrm{Q}$ & $I=10^{\Delta L / 10}$ & $S P=10^{\Delta L / 20}$ & $\mathrm{LF}=10^{\Delta \mathrm{L} / 33.22}=2^{\Delta \mathrm{L} / 10}$ \\
\hline $\mathrm{T} 2$ & 101.5 & 75.1 & 26.4 & 436.5 & 21 & 6.2 \\
\hline T2 FLAIR & 104.4 & 75.9 & 28.5 & 708 & 27 & 7.2 \\
\hline
\end{tabular}

Note:-Avg indicates average; I, intensity factor; SP, sound pressure factor; LF, loudness factor.

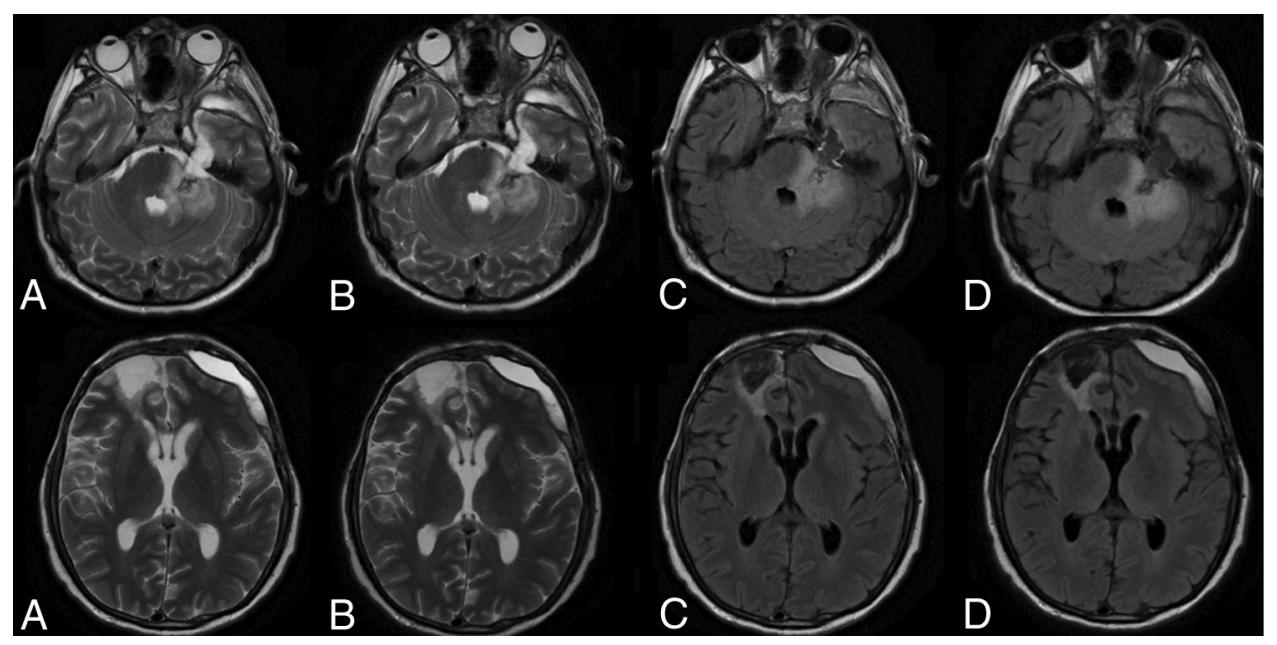

FIG 1. A 58-year-old man with post-craniofacial recurrent tumor resection with postsurgical changes. Comparison of C-T2 (A), Q-T2 (B), C-FLAIR $(C)$, and Q-FLAIR (D). 


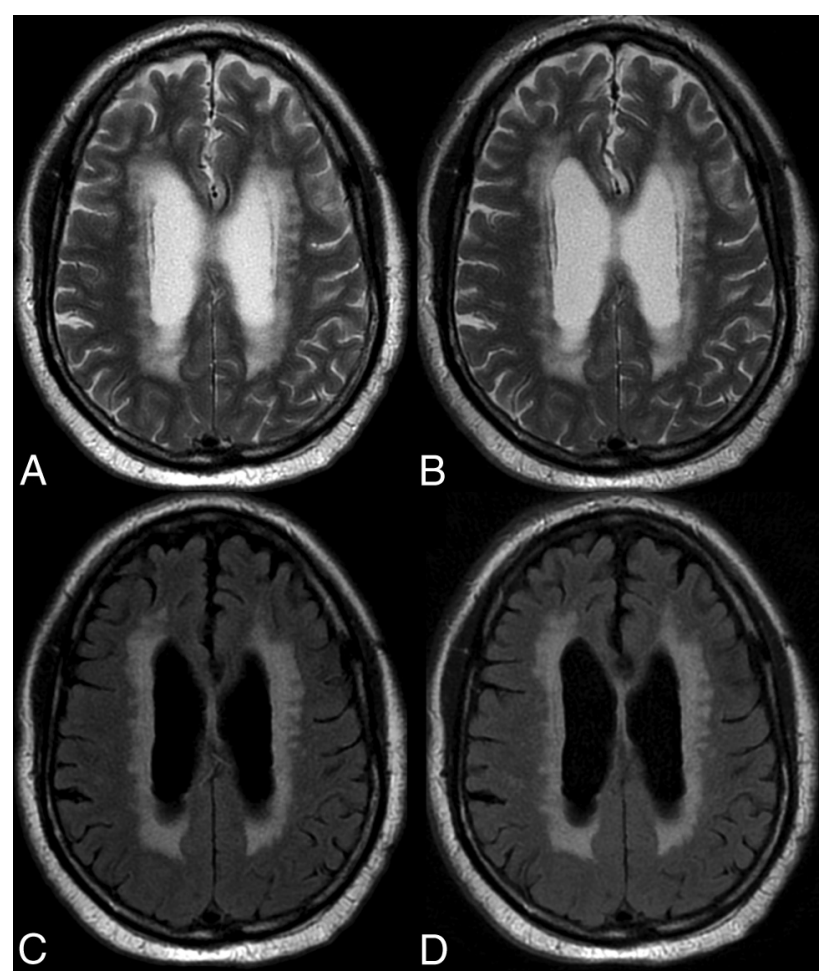

FIG 2. An 82-year-old man with extensive periventricular T2 and T2 FLAIR white matter foci suggesting ischemic small vessel changes. Comparison of C-T2 (A), Q-T2 (B), C-FLAIR (C), and Q-FLAIR (A).

We acknowledge several study limitations. Differences in $k$ space trajectory and overall performance of the quiet techniques required fixed alterations in parameter choices such as TR, echo train, and TE to match spatial resolution and section numbers of the conventional acquisition, potentially contributing to subtle variations in image appearance. While representative of a new class of MR images, these results apply only to the specific approach to noise-reduced imaging and the specific parameter choices made and will not translate exactly to other scanners, techniques, and parameter choices. The small sample size requires a confirmation of our findings in a larger population. As this technology evolves, additional studies will be required in a large population with a variety of disease states.

\section{CONCLUSIONS}

Quiet FSE provides equivalent image quality at comfortable sound pressure levels at the cost of slightly longer scan times. The significant reduction in potentially injurious noise is particularly important in vulnerable populations such as children, the elderly, and the debilitated. Quiet techniques should be used in these special situations and considered for routine use in clinical practice.

\section{ACKNOWLEDGMENTS}

The authors acknowledge Maggie Fung for her assistance.

Disclosures: Idoia Corcuera-Solano_RELATED: Grant: Fundación Alfonso Martín Escudero, Comments: At the time of that research, I was supported by a grant from the Spanish foundation Fundación Alfonso Martín Escudero. Ajeet GaddipatiUNRELATED: Employment: GE Healthcare, Comments: This work was done as part of my employment at GE Healthcare MR Imaging Department; Patents (planned, pend- ing or issued): pending patent through GE Healthcare, Comments: GE Docket No. 265701 (ZPS 8081.704), Comfortable Acoustic Level Techniques for MRI Pulse Sequences. Dawei Gui-UNRELATED: Employment: GE Healthcare; Patents (planned, pending or issued): GE Healthcare, Comments: GE Doc No. 265701-1. Lawrence Tanenbaum—RELATED: Consulting Fee or Honorarium: GE Healthcare; UNRELATED: Payment for Lectures (including service on Speakers Bureaus): GE Healthcare, Siemens.

\section{REFERENCES}

1. Moelker A, Maas RA, Pattynama PM. Verbal communication in MR environments: effect of MR system acoustic noise on speech understanding. Radiology 2004;232:107-13

2. Quirk ME, Letendre AJ, Ciottone RA, et al. Anxiety in patients undergoing MR imaging. Radiology 1989;170:463-66

3. Brummett RE, Talbot JM, Charuhas P. Potential hearing loss resulting from MR imaging. Radiology 1988;169:539-40

4. Radomskij P, Schmidt MA, Heron CW, et al. Effect of MRI noise on cochlear function. Lancet 2002;359:1485

5. McJury M, Shellock FG. Auditory noise associated with MR procedures: a review. J Magn Reson Imaging 2000;12:37-45

6. Reeves MJ, Brandreth M, Whitby EH, et al. Neonatal cochlear function: measurement after exposure to acoustic noise during in utero MR imaging. Radiology 2010;257:802-09

7. Hedeen RA, Edelstein WA. Characterization and prediction of gradient acoustic noise in MR imagers. Magn Reson Med 1997;37:7-10

8. Mansfield P, Haywood B, Coxon R. Active acoustic control in gradient coils for MRI. Magn Reson Med 2001;46:807-18

9. Bowtell RW, Mansfield P. Quiet transverse gradient coils: Lorentz force balanced designs using geometrical similitude. Magn Reson Med 1995;34:494-97

10. Mansfield P, Glover PM, Beaumont J. Sound generation in gradient coil structures for MRI. Magn Reson Med 1998;39:539-50

11. Cho ZH, Chung ST, Chung JY, et al. A new silent magnetic resonance imaging using a rotating DC gradient. Magn Reson Med 1998;39:317-21

12. Segbers M, Rizzo Sierra CV, Duifhuis $H$, et al. Shaping and timing gradient pulses to reduce MRI acoustic noise. Magn Reson Med 2010;64:546-53

13. Hennel F, Girard F, Loenneker T. "Silent" MRI with soft gradient pulses. Magn Reson Med 1999;42:6-10

14. Heismann B, Ott M, Grodzki D. Sequence-based acoustic noise reduction of clinical MRI scans. Magn Reson Med 2015;73:1104-09

15. Pierre EY, Grodzki D, Aandal G, et al. Parallel imaging-based reduction of acoustic noise for clinical magnetic resonance imaging. Invest Radiol 2014;49:620-26

16. Alibek S, Vogel M, Sun W, et al. Acoustic noise reduction in MRI using Silent Scan: an initial experience. Diagn Interv Radiol 2014; 20:360-63

17. Ida M, Wakayama T, Nielsen ML, et al. Quiet T1-weighted imaging using PETRA: initial clinical evaluation in intracranial tumor patients. J Magn Reson Imaging 2015;41:447-53

18. Landis JR, Koch GG. The measurement of observer agreement for categorical data. Biometrics 1977;33:159-74

19. Shellock FG, Morisoli SM, Ziarati M. Measurement of acoustic noise during MR imaging: evaluation of six "worst-case" pulse sequences. Radiology 1994;191:91-93

20. Moelker A, Wielopolski PA, Pattynama PM. Relationship between magnetic field strength and magnetic-resonance-related acoustic noise levels. MAGMA 2003;16:52-55

21. Hurwitz R, Lane SR, Bell RA, et al. Acoustic analysis of gradient-coil noise in MR imaging. Radiology 1989;173:545-48

22. Moelker A, Maas RA, Lethimonnier F, et al. Interventional MR imaging at 1.5 T: quantification of sound exposure. Radiology 2002; 224:889-95

23. Pipe JG, Gibbs WN, Li Z, et al. Revised motion estimation algorithm for PROPELLER MRI. Magn Reson Med 2014;72:430-37 